\title{
Realizing High IPC \\ Using Time-Tagged Resource-Flow Computing
}

\author{
Augustus Uht ${ }^{1}$, Alireza Khalafi ${ }^{2}$, David Morano ${ }^{2}$, \\ Marcos de Alba ${ }^{2}$, and David Kaeli ${ }^{2}$ \\ 1 University of Rhode Island, Kingston, RI, USA, \\ uht@ele.uri.edu \\ 2 Northeastern University, Boston, MA, USA, \\ $\{$ akhalafi,dmorano,mdealba, kaeli\}@ece.neu.edu
}

\begin{abstract}
In this paper we present a novel approach to exploiting ILP through the use of resource-flow computing. This model begins by executing instructions independent of data flow and control flow dependencies in a program. The rest of the execution time is spent applying programmatic data flow and control flow constraints to end up with a programmatically-correct execution. We present the design of a machine that uses time tags and Active Stations, realizing a registerless data path.

In this contribution we focus our discussion on the Execution Window elements of our machine, present Instruction Per Cycle (IPC) speedups for SPECint95 and SPECint2000 programs, and discuss the scalability of our design to hundreds of processing elements.
\end{abstract}

\section{Introduction}

A number of ILP studies have concluded that there exists a significant amount of parallelism in common applications [915,17]. So why haven't we been able to obtain these theoretical speedups? Part of the reason is that we have not been aggressive enough with our execution model.

Lam and Wilson showed us that if a machine could follow multiple flows of control while utilizing a simple branch predictor and limited control dependencies (i.e., instructions after a forward branch's target are independent of the branch), a speedup of 40 could be obtained on average [9. If an Oracle (i.e., perfect) branch predictor was used, speedups averaged 158. Research has already been reported that overcomes many control flow issues using limited multi-path execution 215.

To support rampant speculation while maintaining scalable hardware, we introduce a statically ordered machine that utilizes instruction time tags and Active Stations in the Execution Window. We call our machine Levo [16]. Next we will briefly describe our machine model. 


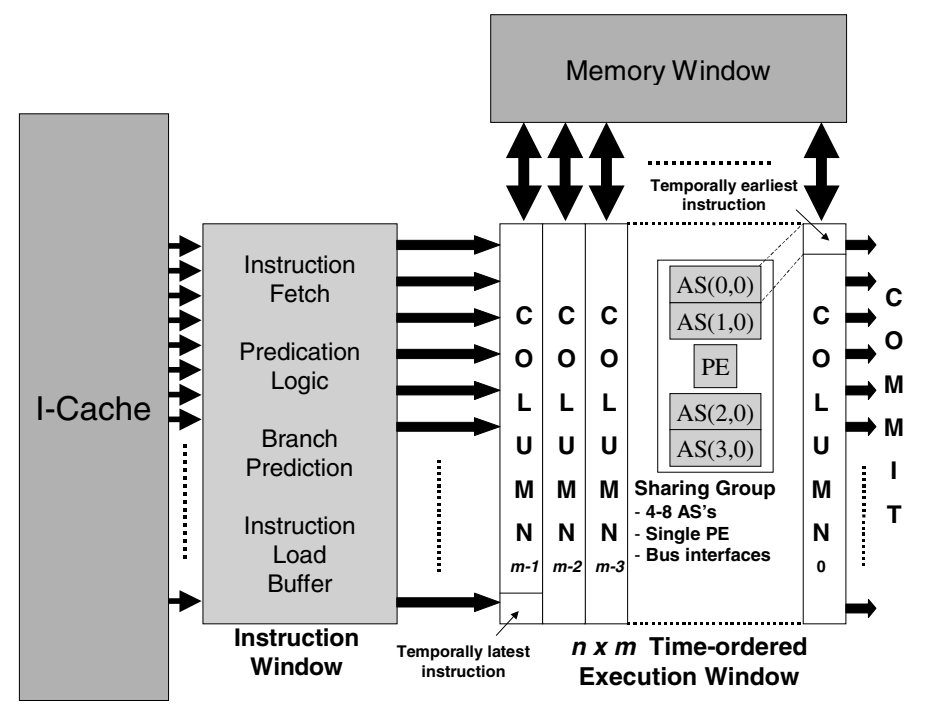

Fig. 1. The Levo machine model.

\section{The Levo Machine Model}

Figure 1 presents the overall model of Levo, which consists of 3 main components: 1) the Instruction Window, 2) the Execution Window, and 3) the Memory Window.

The Instruction Window fetches instructions from an instruction memory, performs dynamic branch prediction, and generates predicates. Instructions are fetched in the static order in which they appear in the binary image (similar to assuming all conditional branches are not taken). By fetching down the not-taken path, we will capture the taken and not taken paths of most branch hammocks 38 . We exploit this opportunity and spawn execution paths to cover both paths (taken and not taken) for hard-to-predict hammocks.

Some exceptions to our static fetch policy are:

1. unconditional jump paths are followed,

2. loops are unrolled dynamically [14] in the Execution Window, and

3 . in the case of conditional branches with far targets, 1 if the branch is strongly predicted taken in the branch predictor, begin static fetching from its target.

We utilize a conventional two-level gshare predictor 11 to guide both instruction fetch (as in case 3 above), as well as to steer instruction issue. Levo utilizes full run-time generated predicates, such that every branch that executes

\footnotetext{
${ }^{1}$ Far implies that the branch target is farther than two-thirds the size of the Execution
} window size. For a machine with 512 ASs, this distance is equal to 341 instructions. 


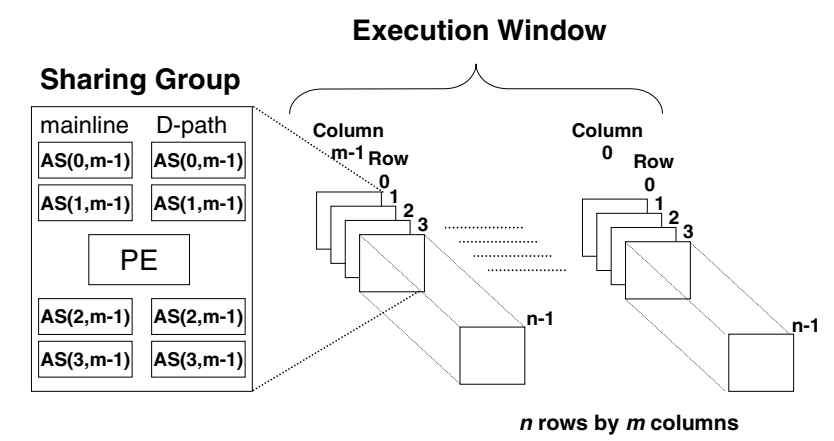

A sharing group of 4 mainline and 4 D-path ASs sharing a single PE

Fig. 2. A Levo Sharing Group.

within the Execution Window (i.e., a branch domain 2), is data and control independent of all other branches.

Levo is an in-order issue, in-order completion machine, though it supports a high degree of speculative resource-flow-order execution. The Execution Window is organized as a grid; columns of processing elements (PEs) are arranged in a number of Sharing Groups (SGs) per column. A SG shares a common PE (see Figure 2).

Levo assigns PEs to the highest priority instruction in a SG that has not been executed, independent of whether the instruction's inputs or operands are known to be correct (data flow independent), and regardless of whether this instruction is known to be on the actual (versus mispredicted) control path (control flow independent). The rest of the execution time is spent applying programmatic data flow (re-executions) and control flow constraints (squashes), so as to end up with a programmatically-correct execution of the program. Instructions are retired in order, when all instructions in the column have completed execution.

Each sharing group contains a number of Active Stations (ASs); instructions are issued in static order to ASs in a column. Each issued instruction is assigned a time tag, based on its location in the column. Time tags play a critical role in the simplicity of Levo by labeling each instruction and operand in our Execution Window. This label is used during the maintenance/enforcement of program order in our highly speculative machine.

Our ASs are designed after Tomasulo's reservation stations [13. There is one instruction per Active Station. Levo ASs are able to snoop and snarf 3 data from buses with the help of the time tags. ASs are also used to evaluate predicates, and to squash redundant operand updates (again using time tags).

${ }^{2}$ A branch domain includes the static instructions starting from the branch to its target, exclusive of the target and the branch itself [15].

${ }^{3}$ Snarfing entails snooping address/data buses, and when the desired address value is detected, the associated data value is read. 
ASs within a Sharing Group compete for the resources of the group, including the single pipelined PE and the broadcast bus outputs. Each spanning bus is connected to adjacent Sharing Groups. The spanning bus length is constant and does not change with the size of the Execution Window; this addresses scalability of this busing structure.

A column in the Execution Window is completely filled with the sequence of instructions as they appear in the Instruction Window. During execution, hardware runtime predication is used for all forward branches with targets within the Execution Window. Backward branches are handled via dynamic loop unrolling [14] and runtime conversion to forward branches.

\subsection{Levo Execution Window Datapath}

Levo's spanning buses play a similar role as Tomasulo's reservation stations' Common Data Bus. Spanning buses are comprised of both forwarding and backwarding buses. Forwarding buses are used to broadcast register, memory and predicate values. If an AS needs an input value, it sends the request to earlier ASs via a backwarding bus and the requested data is returned on a forwarding bus.

An AS connects to the spanning buses corresponding to the position of the AS in the column. Each AS performs simple comparison operations on the time tags and addresses broadcast on the spanning buses to determine whether or not to snarf data or predicates. Figure 3 shows the structure for this function of an AS.

\subsection{Scalability}

So far we have described a machine with ASs all connected together with some small number of spanning buses. In effect, so far there is little difference between a Levo spanning bus and Tomasulo's Common Data Bus. This microarchitecture may reduce the number of cycles needed to execute a program via resource flow, but having the buses go everywhere will increase the cycle time unacceptably.

The Multiscalar project demonstrated that register lifetimes are short, typically spanning only one or two basic blocks (32 instructions at the high end) 15. Based on this important observation, we partition each bus into short segments, limiting the number of ASs connected to any segment; this has been set to the number of ASs in a column for the results presented in this paper.

We interconnect broadcast buses with buffer registers; when a value is transmitted on the bus from the preceding bus segment the sourcing AS needs to compete with other ASs for the bus segment. Local buffer space is provided. Thus, there can be a one or more cycle delay for sending values across bus segments.

In Levo there is no centralized register file, there are no central renaming buffers nor reorder buffer. Levo uses locally-consistent register values distributed throughout the Execution Window and among the PEs. A register's contents are likely to be globally inconsistent, but locally usable. A register's contents will 
eventually become consistent at instruction commit time. In Levo, PEs broadcast their results directly to only a small subset of the instructions in the Execution Window, which includes the instructions within the same Sharing Group.

\subsection{Time Tags and Renaming}

A time tag indicates the position of an instruction in the original sequential program order (i.e., in the order that instructions are issued). ASs are labeled with time tags starting from zero and incrementing up to one minus the total number of ASs in the microarchitecture. A time tag is a small integer that uniquely identifies a particular AS.

Similar to a conventional reservation station, operand results are broadcast forward for use by waiting instructions. With ASs, all operands that are forwarded after the execution of an instruction are also tagged with the time tag value of the AS that generated the updated operand. This tag will be used by subsequent ASs to determine if the operand should be snarfed as an input operand that will trigger the execution of its loaded instruction. Essentially all values within the Execution Window are tagged with time tags. Since our microarchitecture can also allow for the concurrent execution of disjoint paths, we also introduce a path ID.

The microarchitecture that we have devised requires the forwarding of three types of operands. These are register operands, memory operands, and instruction predicate operands. These operands are tagged with time tags and path IDs that are associated with the ASs that produced them. The information broadcast from an AS to subsequent ASs in future program ordered time is referred to as a transaction, and consists of :

- a path ID

- the time tag of the originating AS

- the identifier of the architected operand

- the actual data value for this operand

Figure 3 shows the registers inside an active station for one of its input operands. The time-tag, address, and value registers are reloaded with new values on each snarf, while the path and AS time-tag (column indices) are only loaded when the AS is issued an instruction, with the path register only being reloaded upon a taken disjoint path execution (disjoint execution will be discussed later).

This scheme effectively eliminates the need for rename registers or other speculative registers as part of the reorder buffer. The whole of the microarchitecture thus provides for the full renaming of all operands, thus avoiding all false dependencies. There is no need to limit instruction issue or to limit speculative instruction execution due to a limit on the number of non-architected registers for holding those temporary results. True flow dependencies are enforced through continuous snooping by each AS. 


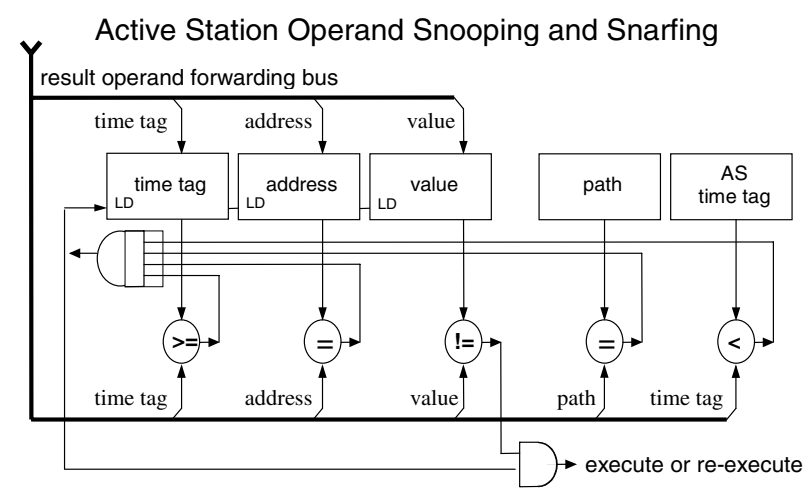

Fig. 3. AS Operand Snooping and Snarfing. The registers and snooping operation of one of several possible source operands is shown. Time tags, addresses and values are compared against each active stations corresponding values. Just one bus is shown being snooped, though several operand forwarding buses are snooped simultaneously.

\subsection{Disjoint Execution}

Our resource flow Execution Window can only produce high IPC if it contains the stream of instructions that will be committed next. In an effort to insure that we can handle the ill-effects of branch mispredictions, we have utilized disjoint execution to handle the cases where branch prediction is wrong.

In Figure 2 we showed a Sharing Group containing both a mainline and disjoint (D-path) set of ASs. The D-path ASs will share the common PE with the mainline execution, though will receive a lower priority when attempting to execute an instruction. The disjoint path is used to hide potential latencies associated with branch mispredictions. The disjoint path is copied from a mainline path in a cycle when the instruction loading buses are free. The disjoint path will use a copy of the mainline path, though will start execution from a point after a branch instruction (if the branch was predicted taken), or at a branch target (if the branch was predicted as not taken) in the mainline execution. For branches that exhibit a chaotic behavior (changing from taken to not-taken often), spawning disjoint paths should be highly beneficial. For more predictable branches (e.g., a loop-ending branch), we can even reap some benefit by executing down the loop exit path.

In [7, we discuss how to select the best path to spawn disjoint paths. In this work, we always start spawning from the column prior to the current column being loaded, and spawn up to 5 paths ( 3 for 4 column configurations). If we look at one of the D-columns, the code and state above the D-branch (the point at which we spawned a disjoint path) is the same as in the mainline path. The code is the same for the entire column (static order). The sign of the predicate of the D-branch is set to the not-predicted direction of the original branch. All other branch predications in the column follow those of the same branches in the mainline column. 
If the D-branch resolves as a correct prediction, the disjoint path state is discarded, and the D-column is reallocated to the next unresolved branch in the mainline. If the $\mathrm{D}$-branch resolves as an incorrect prediction, the mainline state after the D-branch is thrown away, the D-column is renamed as the mainline column, and all other D-column state (for different D-branches) is discarded. Execution resumes with the new mainline state; new D spawning can begin again.

\section{ILP Results}

To evaluate the performance of the ideas we have just described, we have developed a trace-driven simulation model of the Levo machine. The simulator takes as input a trace containing instructions and their associated operand values. We include results for 5 programs taken from the SPECint2000 and SPECint95 suites, using the reference inputs. Our current environment models a MIPS-1 ISA with some MIPS-2 and MIPS-3 instructions included which are used by the SGI compiler or are in SGI system libraries. While we use a specific ISA in this work, Levo is not directly ISA dependent.

For our baseline system (BL), we assume a machine that is bound by true dependencies in the program, and does no forwarding or backwarding of values. The machine follows a single path of execution (no disjoint paths are spawned). We compare the baseline to a variety of Levo systems that implement resource flow (RF). We also show results for a machine that uses D-path spawning (D). We study the effects of different memory systems, assuming both a conventional hierarchical memory system $(\mathrm{CM})$ and a perfect cache memory $(\mathrm{PM})$. All speedup results are relative to a baseline system that uses a conventional memory (BL-CM).

Table 11 summarizes many of the machine parameters we use in the set of results presented. The table includes the parameters for the conventional data memory system. Table 2 shows the 5 different machine configurations studied and presents our baseline IPC numbers which we will use to compare against.

Table 1. Common model simulation parameters.

\begin{tabular}{|c|c|c|}
\hline Feature & Size & Comment \\
\hline \hline Fetch width & 1-column each cycle & \\
\hline L1 I-Cache & & $100 \%$ hit \\
\hline Branch predictor & $\begin{array}{c}\text { 2-level gshare } \\
\text { 1024 PAg 4096 GPHT }\end{array}$ & multi-ported \\
\hline L1 D-Cache & $32 \mathrm{~KB} \mathrm{2-way} \mathrm{32B} \mathrm{line}$ & 4 -way interleaved \\
\hline L1 D-hit time & 1 cycle & \\
\hline L1 D-miss penalty & 10 cycles & \\
\hline L2 and Memory & & $100 \%$ hit \\
\hline Forwarding/Backwarding unit delay & 1 cycle & \\
\hline Bus delay & 1 cycle & \\
\hline
\end{tabular}


parser

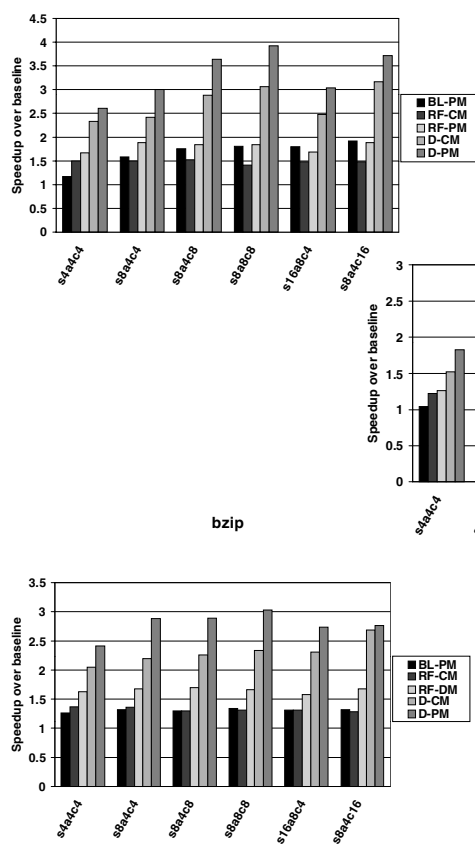

gap

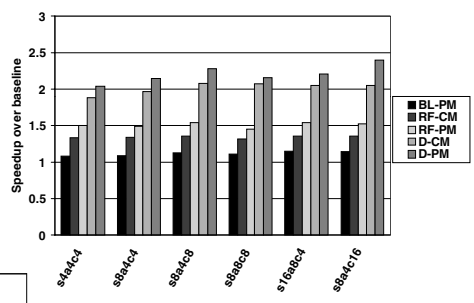

go

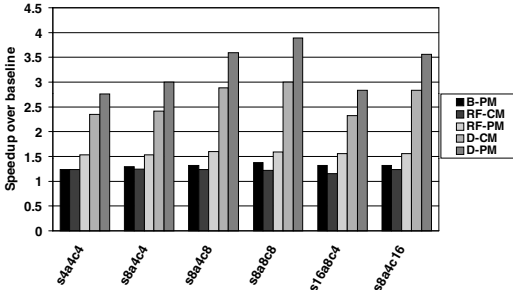

Fig. 4. IPC comparison for baseline perfect memory (BL-PM), resource flow conventional memory (RF-CM), resource flow perfect memory (RF-PM), D-paths conventional memory (D-CM) and D-paths perfect memory (D-PM). All speedup factors are versus our baseline assuming conventional memory (BL-CM).

Table 2. Levo machine configurations and BL-CM IPC values for the 5 benchmarks. $\mathrm{s}=\mathrm{SGs}$ per column, $\mathrm{a}=\mathrm{ASs}$ per $\mathrm{SG}$ and $\mathrm{c}=$ Columns.

\begin{tabular}{|c|c|c|c|c|c|c|c|c|}
\hline $\begin{array}{c}\text { Machine } \\
\text { Config }\end{array}$ & $\begin{array}{l}\text { SGs per } \\
\text { Column }\end{array}$ & $\begin{array}{c}\text { ASs per } \\
\text { SG }\end{array}$ & Columns & $\begin{array}{c}\text { gzip } \\
\text { BL-CM } \\
\text { IPC }\end{array}$ & $\begin{array}{c}\text { gap-CM } \\
\text { IPC }\end{array}$ & $\begin{array}{c}\text { parser } \\
\text { BL-CM } \\
\text { IPC }\end{array}$ & $\begin{array}{c}\text { bzip-CM } \\
\text { IPC }\end{array}$ & $\begin{array}{c}\text { go } \\
\text { IPC-CM }\end{array}$ \\
\hline \hline s4a4c4 & 4 & 4 & 4 & 2.3 & 2.4 & 1.8 & 1.9 & 1.7 \\
\hline s8a4c4 & 8 & 4 & 4 & 2.8 & 3.5 & 2.4 & 2.5 & 2.4 \\
\hline s8a4c8 & 8 & 4 & 8 & 2.9 & 3.9 & 2.5 & 2.7 & 2.5 \\
\hline s8a8c8 & 8 & 8 & 8 & 4.1 & 4.4 & 2.7 & 2.9 & 2.7 \\
\hline s16a8c4 & 16 & 8 & 4 & 3.1 & 3.9 & 2.5 & 2.6 & 2.5 \\
\hline s8a4c16 & 8 & 4 & 16 & 3.1 & 4.2 & 2.5 & 2.5 & 2.5 \\
\hline
\end{tabular}

Figure 4 shows the relative speedup in IPC for our five benchmarks, for the six machine configurations described. All results are relative to our Baseline system with a conventional data cache memory hierarchy, as described in Table 2, The s8a8c8 configuration provides the highest IPC. The first 3 configurations have fewer hardware resources; s16a8c4 does not have enough columns to hide latency 
and while s8a4c16 has enough columns, with fewer ASs per PE, there is lower $\mathrm{PE}$ utilization, and thus a lower IPC is obtained.

While we can see that resource flow provides moderate gains when used alone, we do not see the power of this model until we employ D-paths to hide branch mispredictions. For parser and go, we obtain speedups of 3 to 4 times when using D-paths, with IPCs greater than 10 in 3 out of 5 benchmarks. We should expect go to obtain the most benefit from $\mathrm{D}$ since it possesses the highest percentages of conditional branch mispredictions. Parser shows some performance loss for RF-PM when compared to our baseline. Much of this is due to bus contention, which can be remedied by adding more buses.

\section{Discussion and Summary}

Probably the most successful high-IPC machine to date is Lipasti and Shen's Superspeculative architecture [10, achieving an IPC of about 7 with realistic hardware assumptions. The Ultrascalar machine [6] achieves asymptotic scalability, but only realizes a small amount of IPC, due to its conservative execution model. The Warp Engine 4 uses time tags, like Levo, for a large amount of speculation; however their realization of time tags is cumbersome, utilizing floating point numbers and machine wide parameter updating.

Nagarajan et al. have proposed a Grid Architecture that builds an array of ALUs, each with limited control, connected by a operand network [12]. Their system achieves an IPC of 11 on SPEC2000 and Mediabench benchmarks. While this architecture presents many novel ideas in attempt to reap high IPC, it differs greatly in its interconnect strategy and register design. They also rely on a compiler to obtain this level of IPC, whereas Levo does not.

In this paper we have described the Levo machine model. We have illustrated the power of resource flow and especially D-path execution. We have been successful in obtaining IPCs above 10.

We still believe that there remains substantial ILP to be obtained. In Table 3 we select the configuration that obtained the best D-path result $(8,8,8)$ and show IPC speedup (relative to our D-path result in Figure 4) using an Oracle predictor [9]. As we can see, there still remains a lot of IPC that can be obtained

Table 3. IPC Speedup for Oracle branch prediction for an $8,8,8$ configuration with D-Cache and Perfect Memory. Speedup is relative to the D-CM and D-PM results in Figure 4 .

\begin{tabular}{|c|c|c||}
\hline Benchmark & $\begin{array}{c}\text { IPC Speedup Factor } \\
\text { D-Cache Memory }\end{array}$ & $\begin{array}{c}\text { IPC Speedup Factor } \\
\text { Perfect Memory }\end{array}$ \\
\hline \hline go & 1.6 & 1.8 \\
\hline bzip & 2.1 & 2.4 \\
\hline gzip & 1.5 & 1.6 \\
\hline parser & 1.4 & 1.4 \\
\hline gap & 1.0 & 1.1 \\
\hline
\end{tabular}


through improved control flow speculation. We plan to look at spawning dynamic paths (versus the static path approach described in this work).

\section{References}

1. Austin T.M and Sohi G.S. Dynamic Dependency Analysis of Ordinary Programs. In Proceedings of ISCA-19, pages 342-351, May 1992.

2. Chen T.F. Supporting Highly Speculative Execution via Adapative Branch Trees. In Proceedings of the 4th Annual International Symposium on High Performance Computer Architecture, pages 185-194, January 1998.

3. Cher C.-Y. and Vijaykumar T.N. Skipper: A Microarchitecture For Exploiting Control-Flow Indepdendence. In Proceedings of MICRO-34, December 2001.

4. Cleary J.G, Pearson M.W and Kinawi H. The Architecture of an Optimistic CPU: The Warp Engine. In Proceedings of HICSS, pages 163-172, January 1995.

5. Franklin M. and Sohi G.S. Register Traffic Analysis for Streamlining InterOperation Communication in Fine-Grain Parallel Processors. In Proceedings of MICRO-25, pages 236-247, Dec 1992.

6. Henry D.S and Kuszmaul B.C. and Loh G.H. and Sami R. Circuits for WideWindow Superscalar Processors. In Proceedings of ISCA-27, pages 236-247. ACM, June 2000.

7. Khalafi A., Morano D., Uht A. and Kaeli D. Multipath Execution on a Large-Scale Distributed Microarchitecture. Technical Report 022002-001, University of Rhode Island, Department of Electrical and Computer Engineering, Feb 2002.

8. Klauser A., Austin T., Grunwald D. and Calder B. Dynamic Hammock Predication for Non-Predicated Instruction Set Architectures. In Proceedings of PACT, pages 278-285, 1998.

9. Lam M.S. and Wilson R.P. Limits of Control Flow on Parallelism. In Proceedings of ISCA-19, pages 46-57. ACM, May 1992.

10. Lipasti M.H and Shen J.P. Superarchitecture Microarchitecture for Beyond AD 200. IEEE Computer Magazine, 30(9), September 1997.

11. McFarling S. Combining Branch Predictors. Technical Report DEC WRL TN-36, Digital Equipment Western Research Laboratory, June 1993.

12. Nagarajan R. and Sankaralingam K. and Burger D. and Keckler S. A Design Space Evaluation of Grid Processor Architectures. In Proceedings of MICRO-30, December 2001.

13. Tomasulo R.M. An Efficient Algorithm for Exploiting Multiple Arithmetic Units. IBM Journal of Research and Development, 11(1):25-33, Jan 1967.

14. Tubella J. and Gonzalez A. Control speculation in multithreaded processors through dynamic loop detection. In Proceedings of HPCA-4, pages 14-23, January 1998.

15. Uht, A. K. and Sindagi, V. Disjoint Eager Execution: An Optimal Form of Speculative Execution. In Proceedings of MICRO-28, pages 313-325. ACM-IEEE, November/December 1995.

16. Uht A., Morano D., Khalafi A., Wenisch T., Ashouei M. and Kaeli D. IPC in the 10's via Resource Flow Computing with Levo. Technical Report 092001-001, Unversity of Rhode Island, Department of Electrical and Computer Engineering, Sept 2001.

17. Wall D.W. Limits of Instruction-Level Parallelism. In Proceedings of ASPLOS-4, pages 176-188. ACM, April 1991. 